\title{
Preclinical Alzheimer Disease Drug Development: Early Considerations Based on Phase 3 Clinical Trials
}

\author{
Anna Hung, PharmD, PhD, MS; Monika Schneider, PhD; \\ Marianne Hamilton Lopez, PhD, MPA; and Mark McClellan, MD, PhD
}

\begin{abstract}
SUMMARY
The number of people in the United States living with Alzheimer disease

(AD) is growing, resulting in significant clinical and economic impact. Substantial research investment has led to drug development in stages of $A D$ before symptomatic dementia, such as preclinical AD. Although there are no treatments approved for preclinical AD, there are currently 6 phase 3 clinical trials for preclinical $A D$ treatments. In this article, we review these clinical trials and highlight considerations for future coverage decisions.

In line with the definition of preclinical $A D$, enrollment in these trials focuses on cognitively unimpaired patients that are at high risk of $A D$ because of family history and then genetic testing or brain imaging. Enrollment in most of these trials also allows for younger patients, including those aged under 65 years. Primary clinical trial endpoints focus on cognition often 4 or more years after treatment. Secondary endpoints include other measures of cognition and function, as well as biomarkers.

Review of these trials brings to light a few potential considerations when covering these new medications in the future. First, novel and potentially costly approaches involving genetic testing and/or positron emission tomography imaging may be needed to identify appropriate patients and should be developed efficiently. Second, the long duration of these clinical trials suggest that there may be a need for alternative payment approaches in the United States that encourage early payers to pay for a medication for which the long-term benefits may not be realized until after the beneficiary is no longer with the health plan. Third, the value of $A D$ treatments may differ across populations, creating a potential role for indication-based or population-based contracting. Finally, considering the potentially high budgetary impact and little real-world evidence for a new drug class, payers and manufacturers may want to consider outcomes-based payment approaches and coverage with evidence development to mitigate uncertainty about the value of the treatment demonstrated in well-defined populations in clinical trials versus more heterogeneous real-world settings.
\end{abstract}

J Manag Care Spec Pharm. 2020;26(7):888-900

Copyright $\odot 2020$, Academy of Managed Care Pharmacy. All rights reserved.

A lzheimer disease (AD) is the most common form of dementia, with an estimated prevalence between $10 \%$ and $30 \%$ in the population aged 65 years and above., ${ }^{1,2}$ The cost of health care and long-term care for individuals with $\mathrm{AD}$ or other dementias is high, estimated between $\$ 159$ billion to $\$ 215$ billion in $2010 .^{2}$ These costs are projected to more than double because of the aging of the population by $2040 .^{2}$

There are currently 5 drug products approved by the U.S. Food and Drug Administration (FDA) that are available for the treatment of symptomatic dementia: donepezil, rivastigmine, galantamine, memantine, and a combination drug product with donepezil and memantine. Donepezil and rivastigmine are approved by the FDA for mild, moderate, and severe dementia due to $\mathrm{AD}$; galantamine is approved for mild to moderate dementia due to $\mathrm{AD}$; and memantine is approved for moderate to severe dementia. ${ }^{3-6}$ None of these drugs slow the progression of the disease; instead, they relieve symptoms by increasing the amount of neurotransmitters in the brain. However, in most cases, their efficacy at relieving symptoms is moderate at best and limited in duration, and efficacy varies across patient groups, leaving AD patients and their families with few options for treatment. ${ }^{7-9}$

Reflecting this need and insights into potential new treatment mechanisms, there has been a 5-fold increase in investment to develop drugs that modify disease progression since 2011. ${ }^{10}$ In 2020 alone, the National Institutes of Health are expected to spend $\$ 2.8$ billion on AD research. ${ }^{9}$ Some researchers believe that to slow disease progression and preserve brain function, treatments may be more effective if given earlier in the disease process. ${ }^{11}$ Some of these drugs are currently being developed to target stages of AD before symptomatic dementia.

According to the National Institute on Aging (NIA) and Alzheimer's Association (AA) framework, there are 2 stages before mild, moderate, and severe dementia due to AD: (1) preclinical, when symptoms are not yet present but brain changes have begun, and (2) mild cognitive impairment (MCI) due to AD (also called prodromal AD), which is characterized by brain changes and mild cognitive symptoms that do not significantly affect everyday living. ${ }^{12-14}$ It should be noted that the 2018 update to the NIA-AA framework was intended for research and not routine care, but MCI is diagnosed in routine care based on criteria that were developed in 2011.13,15

If successful and approved for an earlier stage of $\mathrm{AD}$, the drugs that are in development could have a major impact on the care of AD. These medicines could also have substantial effects on the timing and nature of AD-related costs, with significant implications for federal and state programs and commercial insurance. In this article, we review the phase 3 clinical trials for preclinical AD and highlight potential implications for future payer coverage decisions.

Phase 3 Clinical Trials for Preclinical Alzheimer Disease As of 2019, there were 6 phase 3 clinical trials targeting the preclinical stage of $\mathrm{AD}$, with estimated study end dates that 


\section{TABLE 1 Summary of Phase 3 Preclinical Alzheimer Disease Clinical Trials}

\begin{tabular}{|c|c|c|c|c|c|c|}
\hline $\begin{array}{l}\text { Clinical Trial } \\
\text { (estimated } \\
\text { enrollment) } \\
\text { [Sponsor] }\end{array}$ & $\begin{array}{c}\text { Drugs } \\
\text { [Drug Class/Type } \\
\text { (Mechanism of } \\
\text { Action)] }\end{array}$ & Inclusion & Exclusion & $\begin{array}{c}\text { Primary } \\
\text { Endpoint } \\
\text { [Measurement } \\
\text { Time Frame] }\end{array}$ & $\begin{array}{l}\text { Secondary } \\
\text { Endpoints } \\
\text { [Measurement } \\
\text { Time Frame] }\end{array}$ & $\begin{array}{c}\text { Start and } \\
\text { (estimated) } \\
\text { End Date }\end{array}$ \\
\hline \multicolumn{7}{|c|}{ Targeting Preclinical Stage Only } \\
\hline $\begin{array}{l}\text { NCT02008357 } \\
(\mathrm{n}=1,150) \\
\text { [Eli Lilly and } \\
\text { Company }]^{17}\end{array}$ & \begin{tabular}{|l} 
Solanezumab \\
[Monoclonal \\
antibody \\
(anti-amyloid)]
\end{tabular} & $\begin{array}{l}\text { Age } 65-85 \text { years } \\
\text { Has a Mini-Mental State } \\
\text { Examination (MMSE) score } \\
\text { at screening of } 25 \text { to } 30 \text {. } \\
\text { Has a global Clinical } \\
\text { Dementia Rating (CDR) } \\
\text { scale score at screening of } 0 \text {. } \\
\text { Has a Logical Memory II } \\
\text { score at screening of } 6 \text { to } 18 \text {. } \\
\text { Has a florbetapir PET } \\
\text { scan that shows evidence } \\
\text { of brain amyloid pathology } \\
\text { at screening. } \\
\text { Has a study partner that is } \\
\text { willing to participate as a } \\
\text { source of information and } \\
\text { has at least weekly con- } \\
\text { tact with the participant } \\
\text { (contact can be in-person, } \\
\text { via telephone or electronic } \\
\text { communication). }\end{array}$ & $\begin{array}{l}\text { Is receiving a prescription } \\
\text { acetylcholinesterase inhibitor } \\
\text { (AChEI) and/or memantine at } \\
\text { screening or baseline. } \\
\text { Lacks good venous access, } \\
\text { such that intravenous drug } \\
\text { delivery or multiple blood } \\
\text { draws would be precluded. } \\
\text { Has current serious or } \\
\text { unstable illness including } \\
\text { cardiovascular, hepatic, renal, } \\
\text { gastroenterologic, respiratory, } \\
\text { endocrinologic, neurologic, } \\
\text { psychiatric, immunologic, or } \\
\text { hematologic disease or other } \\
\text { conditions that, in the investi- } \\
\text { gator's opinion, could interfere } \\
\text { with the analyses of safety and } \\
\text { efficacy in this study. } \\
\text { Has had a history within the } \\
\text { last } 5 \text { years of a serious infec- } \\
\text { tious disease affecting the brain } \\
\text { (including neurosyphilis, men- } \\
\text { ingitis, or encephalitis) or head } \\
\text { trauma resulting in protracted } \\
\text { loss of consciousness. } \\
\text { Has had a history within the } \\
\text { last } 5 \text { years of a primary or } \\
\text { recurrent malignant disease } \\
\text { with the exception of any in } \\
\text { situ cancer that was appro- } \\
\text { priately treated and is being } \\
\text { appropriately monitored, such } \\
\text { as resected cutaneous squa- } \\
\text { mous cell carcinoma in situ } \\
\text { or in situ prostate cancer with } \\
\text { normal prostate-specific anti- } \\
\text { gen post-treatment. } \\
\text { Has a known history of human } \\
\text { immunodeficiency virus, } \\
\text { clinically significant multiple } \\
\text { or severe drug allergies, } \\
\text { or severe post-treatment } \\
\text { hypersensitivity reactions } \\
\text { (including, but not limited to, } \\
\text { erythema multiforme major, } \\
\text { linear immunoglobulin A } \\
\text { dermatosis, toxic epidermal } \\
\text { necrolysis, or exfoliative } \\
\text { dermatitis). } \\
\text { Is clinically judged by the } \\
\text { investigator to be at serious } \\
\text { risk for suicide. } \\
\text { Has a history within the past } \\
2 \text { years of major depression } \\
\text { or bipolar disorder as defined } \\
\text { by the most current version of } \\
\text { the Diagnostic and Statistical } \\
\text { Manual of Mental Disorders } \\
\text { (DSM). } \\
\text { Has a history within the past } \\
5 \text { years of chronic alcohol or } \\
\text { drug abuse/dependence as } \\
\text { defined by the most current } \\
\text { version of the DSM. }\end{array}$ & $\begin{array}{l}\text { Change from } \\
\text { Baseline of } \\
\text { the Preclinical } \\
\text { Alzheimer } \\
\text { Cognitive } \\
\text { Composite } \\
\text { (PACC) [Time } \\
\text { Frame: Baseline, } \\
\text { Week 240; } \\
\text { Baseline, Week } \\
\text { 336] }\end{array}$ & $\begin{array}{l}\text { 1. Change from } \\
\text { Baseline in Cognitive } \\
\text { Function Index } \\
\text { (CFI) [Time Frame: } \\
\text { Baseline, Week 240; } \\
\text { Baseline, Week 336] } \\
\text { 2. Change from } \\
\text { Baseline in } \\
\text { Alzheimer's Disease } \\
\text { Cooperative Study- } \\
\text { Activities Daily } \\
\text { Living-Prevention } \\
\text { Questionnaire } \\
\text { (ADCS-ADL- } \\
\text { Prevention } \\
\text { Questionnaire) } \\
\text { Score [Time Frame: } \\
\text { Baseline, Week 240; } \\
\text { Baseline, Week 336] } \\
\text { 3. Change from } \\
\text { Baseline in } \\
\text { Mean Composite } \\
\text { Standardized Uptake } \\
\text { Value Ratio (SUVr) } \\
\text { [Time Frame: } \\
\text { Baseline, Week 240] } \\
\text { 4. Change from } \\
\text { Baseline in } \\
\text { Cerebrospinal } \\
\text { Fluid (CSF) Tau } \\
\text { Biomarkers [Time } \\
\text { Frame: Baseline, } \\
\text { Week 240] } \\
\text { 5. Change from } \\
\text { Baseline of CSF } \\
\text { Concentrations } \\
\text { of Amyloid Beta } \\
\text { (Abeta) [Time Frame: } \\
\text { Baseline, Week 240] } \\
\text { 6. Change from } \\
\text { Baseline of } \\
\text { Volumetric Magnetic } \\
\text { Resonance Imaging } \\
\text { (vMRI) [Time Frame: } \\
\text { Baseline, Week 240] } \\
\text { 7. Change from } \\
\text { Baseline on the } \\
\text { Clinical Dementia } \\
\text { Rating-Sum of } \\
\text { Boxes score (CDR-SB) } \\
\text { [Time Frame: } \\
\text { Baseline, Week 336] } \\
\text { 8. Change from } \\
\text { Baseline on the } \\
\text { Computerized } \\
\text { Cognitive Composite } \\
\text { (C3) [Time Frame: } \\
\text { Baseline, Week 336] }\end{array}$ & $\begin{array}{l}\text { February } \\
2014 \& \\
\text { July } 2022\end{array}$ \\
\hline
\end{tabular}




\section{TABLE 1 Summary of Phase 3 Preclinical Alzheimer Disease Clinical Trials (continued)}

\begin{tabular}{|c|c|c|c|c|c|c|}
\hline $\begin{array}{l}\text { Clinical Trial } \\
\text { (estimated } \\
\text { enrollment) } \\
\text { [Sponsor] }\end{array}$ & $\begin{array}{c}\text { Drugs } \\
\text { [Drug Class/Type } \\
\text { (Mechanism of } \\
\text { Action)] }\end{array}$ & Inclusion & Exclusion & $\begin{array}{c}\text { Primary } \\
\text { Endpoint } \\
\text { [Measurement } \\
\text { Time Frame] }\end{array}$ & $\begin{array}{l}\text { Secondary } \\
\text { Endpoints } \\
\text { [Measurement } \\
\text { Time Frame] }\end{array}$ & $\begin{array}{c}\text { Start and } \\
\text { (estimated) } \\
\text { End Date }\end{array}$ \\
\hline $\begin{array}{l}\text { NCT02565511a } \\
(\mathrm{n}=481) \\
{[\text { Novartis }]^{18}}\end{array}$ & $\begin{array}{l}\text { CAD106, } \\
\text { CNP520 } \\
\text { [Amyloid } \\
\text { vaccine and } \\
\text { BACE inhibitor } \\
\text { (anti-amyloid)] }\end{array}$ & $\begin{array}{l}\text { Consent to receive disclo- } \\
\text { sure of their risk estimates } \\
\text { to develop clinical symp- } \\
\text { toms of AD based on their } \\
\text { APOE genotype. } \\
\text { Male or female, age } 60 \text { to } \\
75 \text { years inclusive. Females } \\
\text { must be considered post- } \\
\text { menopausal and not of } \\
\text { child bearing potential. } \\
\text { Mini-Mental State } \\
\text { Examination (MMSE) total } \\
\text { score } \geq 24 \text { (at screening or } \\
\text { in previous } 3 \text { months) and } \\
\text { cognitively unimpaired as } \\
\text { evaluated by memory tests } \\
\text { performed at screening. } \\
\text { Homozygous APOE4 } \\
\text { genotype. } \\
\text { Participant's willingness to } \\
\text { have a study partner. }\end{array}$ & $\begin{array}{l}\text { Any disability that may prevent } \\
\text { the participants from complet- } \\
\text { ing all study requirements. } \\
\text { Current medical or neurologi- } \\
\text { cal condition that might impact } \\
\text { cognition or performance on } \\
\text { cognitive assessments. } \\
\text { Advanced, severe progressive } \\
\text { or unstable disease that may } \\
\text { interfere with the safety, toler- } \\
\text { ability and study assessments, } \\
\text { or put the participant at spe- } \\
\text { cial risk. } \\
\text { History of malignancy of } \\
\text { any organ system, treated or } \\
\text { untreated, within the past } 60 \\
\text { months. } \\
\text { History of hypersensitivity } \\
\text { to any of the investigational } \\
\text { drugs or their excipients/ } \\
\text { adjuvant or to drugs of similar } \\
\text { chemical classes. } \\
\text { Indication for, or current } \\
\text { treatment with ChEIs and/or } \\
\text { another AD treatment } \\
\text { (e.g., memantine). } \\
\text { Contraindication or intoler- } \\
\text { ance to MRI or PET investiga- } \\
\text { tions (with fluorinated radio } \\
\text { ligands). } \\
\text { Brain MRI results showing } \\
\text { findings unrelated to AD } \\
\text { that, in the opinion of the } \\
\text { Investigator might be a lead- } \\
\text { ing cause to future cognitive } \\
\text { decline, might pose a risk to } \\
\text { the participant, or might pre- } \\
\text { vent a satisfactory MRI assess- } \\
\text { ment for safety monitoring. } \\
\text { Suicidal Ideation in the past } 6 \\
\text { months, or Suicidal Behavior } \\
\text { in the past } 2 \text { years. } \\
\text { A positive drug screen at } \\
\text { Screening, if, in the investiga- } \\
\text { tor's opinion, this is due to } \\
\text { drug abuse. } \\
\text { Significantly abnormal labora- } \\
\text { tory results at Screening, or } \\
\text { infection not as a result of a } \\
\text { temporary condition. } \\
\text { Current clinically significant } \\
\text { ECG findings. } \\
\text { For Cohort - I only: } \\
\text { Participants with previous } \\
\text { organ transplantation or stem } \\
\text { cell transplantation, or indica- } \\
\text { tion for treatment with anti- } \\
\text { coagulants. } \\
\text { For Cohort - II only: } \\
\text { Participants with depigment- } \\
\text { ing or hypopigmenting condi- } \\
\text { tions (e.g., albinism vitiligo) } \\
\text { or active/history of chronic } \\
\text { urticarial in the past year. }\end{array}$ & $\begin{array}{l}\text { Time to } \\
\text { diagnosis of } \\
\text { MCI due to AD } \\
\text { or dementia } \\
\text { due to AD } \\
\text { [Time Frame: } \\
\text { Through study } \\
\text { completion, an } \\
\text { average of } 5 \\
\text { years] } \\
\text { Change in the } \\
\text { Alzheimer's } \\
\text { Prevention } \\
\text { Initiative } \\
\text { Composite } \\
\text { Cognitive } \\
\text { (APCC) Test } \\
\text { Score [Time } \\
\text { Frame: Baseline } \\
\text { to Month 60] }\end{array}$ & $\begin{array}{l}\text { 1. Change in Clinical } \\
\text { Dementia Rating } \\
\text { Scale Sum of Boxes } \\
\text { (CDR-SOB) score } \\
\text { [Time Frame: } \\
\text { Baseline to Month 60] } \\
\text { 2. Number of partici- } \\
\text { pants with Adverse } \\
\text { Events as a mea- } \\
\text { sure of Safety and } \\
\text { Tolerability [Time } \\
\text { Frame: Through } \\
\text { study completion, an } \\
\text { average of } 5 \text { years] } \\
\text { 3. Change on the } \\
\text { Total Scale score } \\
\text { and individual neu- } \\
\text { rocognitive domain } \\
\text { index scores of the } \\
\text { Repeatable Battery } \\
\text { for the Assessment of } \\
\text { Neuropsychological } \\
\text { Status (RBANS) [Time } \\
\text { Frame: Baseline to } \\
\text { Month 60] } \\
\text { 4. Change in the } \\
\text { Everyday Cognition } \\
\text { scale (ECog) total } \\
\text { scores [Time Frame: } \\
\text { Baseline to Month 60] } \\
\text { 5. Change in } \\
\text { AD-related biomark- } \\
\text { ers [Time Frame: } \\
\text { Baseline to Months } \\
\text { 24 and 60] } \\
\text { 6. Change in APCC } \\
\text { Test Score [Time } \\
\text { Frame: Month 6 to } \\
\text { Month 60] } \\
\text { 7. Change in CDR- } \\
\text { SOB [Time Frame: } \\
\text { Month } 6 \text { to Month 60] } \\
\text { 8. A beta-specific } \\
\text { immune response } \\
\text { [Time Frame: } \\
\text { Through study com- } \\
\text { pletion, an average } \\
\text { of } 5 \text { years] }\end{array}$ & $\begin{array}{l}\text { November } \\
2015 \& \\
\text { March } 2025\end{array}$ \\
\hline
\end{tabular}




\section{TABLE 1 Summary of Phase 3 Preclinical Alzheimer Disease Clinical Trials (continued)}

\begin{tabular}{|c|c|c|c|c|c|c|}
\hline $\begin{array}{l}\text { Clinical Trial } \\
\text { (estimated } \\
\text { enrollment) } \\
\text { [Sponsor] }\end{array}$ & $\begin{array}{c}\text { Drugs } \\
\text { [Drug Class/Type } \\
\text { (Mechanism of } \\
\text { Action)] }\end{array}$ & Inclusion & Exclusion & $\begin{array}{c}\text { Primary } \\
\text { Endpoint } \\
\text { [Measurement } \\
\text { Time Frame] }\end{array}$ & $\begin{array}{l}\text { Secondary } \\
\text { Endpoints } \\
\text { [Measurement } \\
\text { Time Frame] }\end{array}$ & $\begin{array}{c}\text { Start and } \\
\text { (estimated) } \\
\text { End Date }\end{array}$ \\
\hline $\begin{array}{l}\text { NCT03131453a } \\
(n=1,145) \\
{[\text { Novartis }]^{19}}\end{array}$ & $\begin{array}{l}\text { CNP520 } \\
\text { [BACE inhibitor } \\
\text { (anti-amyloid)] }\end{array}$ & $\begin{array}{l}\text { Consent to receive disclo- } \\
\text { sure of their risk estimates } \\
\text { to develop clinical symp- } \\
\text { toms of AD based on their } \\
\text { APOE genotype and, if } \\
\text { Heterozygotes, evidence of } \\
\text { elevated brain amyloid. } \\
\text { Male or female, age } 60 \text { to } \\
75 \text { years inclusive. Females } \\
\text { must be considered post- } \\
\text { menopausal and not of } \\
\text { child bearing potential. } \\
\text { Cognitively unimpaired as } \\
\text { evaluated by memory tests } \\
\text { performed at screening. } \\
\text { Participant's willingness to } \\
\text { have a study partner. } \\
\text { Carrier of at least } \\
\text { one APOE4 gene if } \\
\text { Heterozygotes, elevated } \\
\text { brain amyloid (as mea- } \\
\text { sured by CSF Abeta or } \\
\text { amyloid PET imaging). }\end{array}$ & $\begin{array}{l}\text { Any disability that may prevent } \\
\text { the participants from complet- } \\
\text { ing all study requirements. } \\
\text { Current medical or neurologi- } \\
\text { cal condition that might impact } \\
\text { cognition or performance on } \\
\text { cognitive assessments. } \\
\text { Advanced, severe progressive } \\
\text { or unstable disease that may } \\
\text { interfere with the safety, toler- } \\
\text { ability and study assessments, } \\
\text { or put the participant at spe- } \\
\text { cial risk. } \\
\text { History of malignancy of } \\
\text { any organ system, treated or } \\
\text { untreated, within the past } 60 \\
\text { months. } \\
\text { Indication for, or current } \\
\text { treatment with, ChEIs and/ } \\
\text { or another AD treatment (e.g., } \\
\text { memantine). } \\
\text { Contraindication or intoler- } \\
\text { ance to MRI. } \\
\text { Brain MRI results showing } \\
\text { findings unrelated to AD } \\
\text { that, in the opinion of the } \\
\text { Investigator might be a lead- } \\
\text { ing cause to future cognitive } \\
\text { decline, might pose a risk to } \\
\text { the participant, or might pre- } \\
\text { vent a satisfactory MRI assess- } \\
\text { ment for safety monitoring. } \\
\text { Suicidal Ideation in the past } 6 \\
\text { months, or Suicidal Behavior } \\
\text { in the past } 2 \text { years. } \\
\text { A positive drug screen } \\
\text { at Screening, if, in the } \\
\text { Investigator's opinion, this is } \\
\text { due to drug abuse. } \\
\text { Significantly abnormal labo- } \\
\text { ratory results at Screening, } \\
\text { not as a result of a temporary } \\
\text { condition. } \\
\text { Current clinically significant } \\
\text { ECG findings. } \\
\text { Clinically relevant depigment- } \\
\text { ing or hypopigmenting condi- } \\
\text { tions (e.g., albinism, vitiligo) } \\
\text { or active/history of chronic } \\
\text { urticaria in the past year. }\end{array}$ & $\begin{array}{l}\text { 1. Time to event } \\
\text { [Time Frame: } \\
\text { Through study } \\
\text { completion, at } \\
\text { least } 5 \text { years], } \\
\text { where event is } \\
\text { defined as diag- } \\
\text { nosis of MCI } \\
\text { due to AD or } \\
\text { dementia due to } \\
\text { AD, whichever } \\
\text { occurs first dur- } \\
\text { ing the course of } \\
\text { the study, after } \\
\text { confirmation by } \\
\text { the adjudication } \\
\text { committee } \\
\text { 2. Change in } \\
\text { the Alzheimer's } \\
\text { Prevention } \\
\text { Initiative } \\
\text { Composite } \\
\text { Cognitive } \\
\text { (APCC) Test } \\
\text { Score [Time } \\
\text { Frame: Baseline } \\
\text { to Month 60] }\end{array}$ & $\begin{array}{l}\text { 1. Change in } \\
\text { Clinical Dementia } \\
\text { Rating Scale Sum of } \\
\text { Boxes (CDR-SOB) } \\
\text { score [Time Frame: } \\
\text { Baseline to Month 60] } \\
\text { 2. Change on the } \\
\text { Total Scale score } \\
\text { and individual } \\
\text { neurocognitive } \\
\text { domain index } \\
\text { scores of the } \\
\text { Repeatable Battery } \\
\text { for the Assessment of } \\
\text { Neuropsychological } \\
\text { Status (RBANS) [Time } \\
\text { Frame: Baseline to } \\
\text { Month 60] } \\
\text { 3. Change in the } \\
\text { Everyday Cognition } \\
\text { scale (ECog) total } \\
\text { scores [Time Frame: } \\
\text { Baseline to Month 60] } \\
\text { 4. Change in cerebral } \\
\text { amyloid angiopathy } \\
\text { (CAA) [Time Frame: } \\
\text { Through study } \\
\text { completion, at least } \\
5 \text { years] } \\
\text { 5. Change on volume } \\
\text { of brain regions } \\
\text { [Time Frame: } \\
\text { Baseline to Month 60] } \\
\text { 6. Change in } \\
\text { amyloid deposition } \\
\text { as measured by } \\
\text { standardized uptake } \\
\text { ratio (SUVR) of PET } \\
\text { scan with amyloid } \\
\text { radiotracer [Time } \\
\text { Frame: Baseline to } \\
\text { Months } 24 \text { and 60] } \\
\text { 7. Change in CSF } \\
\text { levels of A } 340, \\
\text { Aß42 [Time Frame: } \\
\text { Baseline to Months } \\
\text { 24 and 60] } \\
\text { 8. Change in CSF } \\
\text { levels of total tau } \\
\text { and phosphorylated } \\
\text { tau [Time Frame: } \\
\text { Baseline to Months } \\
\text { 24 and 60] } \\
\text { 9. Number of partici- } \\
\text { pants with adverse } \\
\text { events as a measure } \\
\text { of safety [Time } \\
\text { Frame: Through } \\
\text { study completion, at } \\
\text { least } 5 \text { years] }\end{array}$ & $\begin{array}{l}\text { August } 2017 \\
\& \text { March } \\
2025^{b}\end{array}$ \\
\hline
\end{tabular}




\section{TABLE 1 Summary of Phase 3 Preclinical Alzheimer Disease Clinical Trials (continued)}

\begin{tabular}{|c|c|c|c|c|c|c|}
\hline $\begin{array}{l}\text { Clinical Trial } \\
\text { (estimated } \\
\text { enrollment) } \\
\text { [Sponsor] }\end{array}$ & $\begin{array}{c}\text { Drugs } \\
\text { [Drug Class/Type } \\
\text { (Mechanism of } \\
\text { Action)] }\end{array}$ & Inclusion & Exclusion & $\begin{array}{c}\text { Primary } \\
\text { Endpoint } \\
\text { [Measurement } \\
\text { Time Frame] }\end{array}$ & $\begin{array}{l}\text { Secondary } \\
\text { Endpoints } \\
\text { [Measurement } \\
\text { Time Frame] }\end{array}$ & $\begin{array}{l}\text { Start and } \\
\text { (estimated) } \\
\text { End Date }\end{array}$ \\
\hline & & & & & $\begin{array}{l}\text { 10. Change in neuro- } \\
\text { fibrillary tangle bur- } \\
\text { den as measured by } \\
\text { standardized uptake } \\
\text { ratio (SUVR) of PET } \\
\text { scans with tau radio- } \\
\text { tracer (where avail- } \\
\text { able) [Time Frame: } \\
\text { Baseline to Months } \\
24 \text { and } 60 \text { ] }\end{array}$ & \\
\hline $\begin{array}{l}\text { NCT02719327a } \\
\text { (n= 150) } \\
\text { [VA Office of } \\
\text { Research and } \\
\text { Development] }\end{array}$ & $\begin{array}{l}\text { Icosapent ethyl } \\
\text { [Purified form } \\
\text { of omega-3 fatty } \\
\text { acid EPA (neuro- } \\
\text { protection)] }\end{array}$ & $\begin{array}{l}\text { U.S Veteran eligible for VA } \\
\text { care. } \\
\text { Age } 50-75 \text { years, inclusive. } \\
\text { Cognitively healthy. } \\
\text { With increased risk for } \\
\text { developing AD due to } \\
\text { parental history of the dis- } \\
\text { ease and increased preva- } \\
\text { lence of APOE4 allele.c }\end{array}$ & $\begin{array}{l}\text { Dementia or mild cognitive } \\
\text { impairment on screening } \\
\text { evaluation. } \\
\text { Current use of fish oil supple- } \\
\text { ments (requires } 3 \text { month } \\
\text { wash-out period). } \\
\text { Active liver disease with AST } \\
\text { or ALT greater than twice the } \\
\text { upper limit of normal. } \\
\text { Elevated creatine kinase } \\
\text { greater than twice the upper } \\
\text { limit of normal. } \\
\text { Prior adverse reaction to } \\
\text { statins or fish oil. } \\
\text { Pregnant, nursing, or preg- } \\
\text { nancy planned. } \\
\text { Use of medications that inter- } \\
\text { act with icosapent ethyl. } \\
\text { Current use of anticoagulants. } \\
\text { Known hypersensitivity to fish } \\
\text { and/or shellfish. } \\
\text { Current use of other investiga- } \\
\text { tional drug. } \\
\text { History of significant athero- } \\
\text { sclerotic cardiovascular disease } \\
\text { or diabetes mellitus. } \\
\text { Low-density lipoprotein } \\
\text { cholesterol } \geq 190 \text { mg/dL or } \\
<80 \text { mg/dL. } \\
\text { Triglycerides } \geq 500 \text { mg/dL. } \\
\text { Creatinine }>1.8 \text { mg/dL. } \\
\text { Previous lumbar surgery with } \\
\text { contraindication to lumbar } \\
\text { puncture. } \\
\text { Claustrophobia requiring seda- } \\
\text { tion for MRI. } \\
\text { Pacemaker or other contraindi- } \\
\text { cation for MRI. } \\
\text { Consumption of }>200 \text { mg per } \\
\text { day omega-3 fatty acids in diet. }\end{array}$ & $\begin{array}{l}\text { Regional cerebral } \\
\text { blood flow using } \\
\text { arterial spin- } \\
\text { labeling MRI } \\
\text { [Time Frame: } \\
18 \text { month ] }\end{array}$ & $\begin{array}{l}\text { Cerebrospinal fluid } \\
\text { (CSF) biomarkers of } \\
\text { Alzheimer's disease } \\
\text { [Time Frame: } 18 \\
\text { months], including } \\
\text { CSF beta-amyloid, } \\
\text { total tau, and phos- } \\
\text { phorylated tau } \\
\text { Cognitive perfor- } \\
\text { mance [Time Frame: } \\
18 \text { months], mea- } \\
\text { sured by Preclinical } \\
\text { Alzheimer's } \\
\text { Cognitive Composite } \\
\text { (PACC) }\end{array}$ & $\begin{array}{l}\text { June } 2017 \text { \& } \\
\text { November } \\
2021\end{array}$ \\
\hline $\begin{array}{l}\text { NCT02913664a } \\
(\mathrm{n}=640) \\
\text { [University } \\
\text { of Texas } \\
\text { Southwestern } \\
\text { Medical Center] }{ }^{21}\end{array}$ & $\begin{array}{l}\text { Losartan, } \\
\text { amlodipine, } \\
\text { atorvastatin } \\
\text { [Angiotensin II } \\
\text { receptor blocker, } \\
\text { calcium chan- } \\
\text { nel blocker, } \\
\text { cholesterol agent } \\
\text { (neuroprotec- } \\
\text { tion through } \\
\text { improvement in } \\
\text { cardiovascular } \\
\text { health)] }\end{array}$ & $\begin{array}{l}\text { 1. Age } 60-85 \text {, all races/eth- } \\
\text { nicities, and both sexes are } \\
\text { eligible. } \\
\text { 2. a) A positive family his- } \\
\text { tory of dementia defined } \\
\text { as having at least one first- } \\
\text { degree relative with a his- } \\
\text { tory of AD or other type of } \\
\text { dementia, or b) having sub- } \\
\text { jective cognitive decline. }\end{array}$ & $\begin{array}{l}\text { 1. Clinically documented } \\
\text { history of stroke, focal neu- } \\
\text { rological signs or other major } \\
\text { cerebrovascular diseases based } \\
\text { on clinical judgment or MRI/ } \\
\text { CT scans such as evidence of } \\
\text { infection, infarction or other } \\
\text { brain lesions. }\end{array}$ & $\begin{array}{l}\text { Change in global } \\
\text { neurocognitive } \\
\text { function }\end{array}$ & $\begin{array}{l}\text { 1. Domain-specific } \\
\text { neurocognitive } \\
\text { function assessed } \\
\text { by using the tests } \\
\text { included in the } \\
\text { ADCS-PACC and } \\
\text { NIH-TB Cognition. } \\
\text { [Time Frame: } 2 \text { Years] }\end{array}$ & $\begin{array}{l}\text { September } \\
2016 \& \\
\text { September } \\
2022\end{array}$ \\
\hline
\end{tabular}




\section{TABLE 1 Summary of Phase 3 Preclinical Alzheimer Disease Clinical Trials (continued)}

\begin{tabular}{|c|c|c|c|c|c|c|}
\hline $\begin{array}{l}\text { Clinical Trial } \\
\text { (estimated } \\
\text { enrollment) } \\
\text { [Sponsor] }\end{array}$ & $\begin{array}{c}\text { Drugs } \\
\text { [Drug Class/Type } \\
\text { (Mechanism of } \\
\text { Action)] }\end{array}$ & Inclusion & Exclusion & $\begin{array}{c}\text { Primary } \\
\text { Endpoint } \\
\text { [Measurement } \\
\text { Time Frame] }\end{array}$ & $\begin{array}{l}\text { Secondary } \\
\text { Endpoints } \\
\text { [Measurement } \\
\text { Time Frame] }\end{array}$ & $\begin{array}{c}\text { Start and } \\
\text { (estimated) } \\
\text { End Date }\end{array}$ \\
\hline & & $\begin{array}{l}\text { 3. Mini-Mental State Exam } \\
\text { (MMSE) } \geq 26 \text { to exclude } \\
\text { gross dementia. } \\
\text { 4. Must lead a sedentary } \\
\text { lifestyle defined by not } \\
\text { having an "active" rating } \\
\text { on Rapid Assessment of } \\
\text { Physical Activity (RAPA), } \\
\text { i.e., score below } 6 \text { on RAPA. } \\
\text { 5. a) Individuals treated for } \\
\text { HTN with } 110 \leq \text { SBP } \leq 130 \\
\text { mmHg; or b) Individuals } \\
\text { with SBP }>130 \text { and } \\
\text { SBP }<180 \text { (If an individual, } \\
\text { not treated for HTN, has a } \\
\text { SBP } \geq 125 \text { mmHg, consider } \\
\text { rescreening after } 24 \text { hours). } \\
\text { 6. Willingness to be ran- } \\
\text { domized into the treatment } \\
\text { groups and ability to return } \\
\text { to clinic for follow-up visits } \\
\text { over } 24 \text { months. } \\
\text { 7. Fluency in English, ade- } \\
\text { quate visual and auditory } \\
\text { acuity to allow neuropsy- } \\
\text { chological testing. } \\
\text { 8. Participants must have a } \\
\text { regular health care provider. } \\
\text { 9. Physical ability to } \\
\text { undergo exercise training; } \\
\text { able to walk } 10 \text { minutes } \\
\text { without pain. }\end{array}$ & $\begin{array}{l}\text { 2. Diagnosis of AD or other } \\
\text { type of dementia, or signifi- } \\
\text { cant neurologic diseases such } \\
\text { as Parkinson disease, seizure } \\
\text { disorder, multiple sclerosis, } \\
\text { history of severe head trauma } \\
\text { or normal pressure hydro- } \\
\text { cephalus. } \\
\text { 3. Evidence of severe major } \\
\text { depression (GDS }>12 \text {, may be } \\
\text { rescreened after } 12 \text { weeks or } \\
\text { longer if evidence of reactive } \\
\text { depression or temporary mood } \\
\text { disturbances or clinically sig- } \\
\text { nificant psychopathology (e.g., } \\
\text { psychosis and schizophrenia); } \\
\text { if hospitalized in past year, can } \\
\text { be rescreened in } 6 \text { months; or } \\
\text { presence of a major psychiatric } \\
\text { disorder that in the investiga- } \\
\text { tor's opinion, could interfere } \\
\text { with adherence to research } \\
\text { assessments or procedures. } \\
\text { 4. Unstable heart disease based } \\
\text { on clinical judgment (e.g., } \\
\text { heart attack/cardiac arrest, car- } \\
\text { diac bypass procedures within } \\
\text { previous } 6 \text { months and con- } \\
\text { gestive heart failure) or other } \\
\text { severe medical conditions. } \\
\text { 5. History of atrial fibrillation } \\
\text { and evidence on ECG with } \\
\text { any of the following: active } \\
\text { symptoms of persistent pal- } \\
\text { pitation, dizziness, history of } \\
\text { syncope, chest pain, dyspnea, } \\
\text { orthopnea, shortness of breath } \\
\text { at rest, or paroxysmal noctur- } \\
\text { nal dyspnea within the past } 6 \\
\text { months; resting heart rate of }< \\
\text { 30 or > } 110 \text { bpm; taking class } \\
\text { I or III anti-arrhythmic drugs } \\
\text { including flecanide, propafe- } \\
\text { none, dronedarone, sotalol, } \\
\text { dofetilide, and amiodarone; } \\
\text { or clinical concerns for safely } \\
\text { participating in exercise and } \\
\text { lowering blood pressure. } \\
\text { 6. Systolic BP equal or greater } \\
\text { than 180 mmHg and/or dia- } \\
\text { stolic BP equal or greater than } \\
\text { 110 mmHg, may be rescreened } \\
\text { in } 1 \text { week. } \\
\text { 7. Orthostatic hypotension, } \\
\text { defined as the third stand-- } \\
\text { ing SBP }<100 \text { mmHg, may be } \\
\text { rescreened after } 2 \text { weeks. } \\
\text { 8. History of significant auto- } \\
\text { immune disorders such as } \\
\text { systemic lupus erythematosus, } \\
\text { rheumatoid arthritis, or poly- } \\
\text { myalgia rheumatic. } \\
\text { 9. Significant history of alco- } \\
\text { holism or drug abuse within } \\
\text { the last } 5 \text { years. }\end{array}$ & $\begin{array}{l}\text { [Time Frame: } \\
2 \text { Years], as } \\
\text { measured by } \\
\text { Alzheimer's } \\
\text { Disease } \\
\text { Cooperative } \\
\text { Study- } \\
\text { Preclinical } \\
\text { Alzheimer } \\
\text { Cognitive } \\
\text { Composite } \\
\text { (ADCS-PACC) } \\
\text { and NIH } \\
\text { Toolbox } \\
\text { (NIH-TB) } \\
\text { Cognition } \\
\text { Battery } \\
\end{array}$ & $\begin{array}{l}\text { 2. Whole brain and } \\
\text { hippocampal volume } \\
\text { assessed via Magnetic } \\
\text { Resonance Imaging } \\
\text { (MRI). [Time Frame: } \\
\text { 2 years] } \\
\text { 3. Global and } \\
\text { regional brain per- } \\
\text { fusion assessed via } \\
\text { MRI. [Time Frame: } \\
\text { 2 years] } \\
\text { 4. Brain white mat- } \\
\text { ter hyperintensity } \\
\text { (WMH) assessed via } \\
\text { MRI. [Time Frame: } \\
2 \text { years] } \\
\text { 5. Brain white mat- } \\
\text { ter microstructural } \\
\text { integrity assessed via } \\
\text { MRI. [Time Frame: } \\
\text { 2 years] } \\
\text { 6. Brain neural } \\
\text { network functional } \\
\text { connectivity assessed } \\
\text { via functional MRI. } \\
\text { [Time Frame: } 2 \text { years] } \\
\text { 7. Patient-reported } \\
\text { outcomes (PRO) of } \\
\text { mental and physical } \\
\text { health and health- } \\
\text { related quality of life } \\
\text { assessed by using } \\
\text { NIH PROMIS. [Time } \\
\text { Frame: } 2 \text { years] } \\
8 \text {. Physical func- } \\
\text { tion assessed via } \\
\text { the Short Physical } \\
\text { Performance Battery } \\
\text { (SPPB). [Time Frame: } \\
2 \text { years] } \\
\text { 9. Dual task perfor- } \\
\text { mance assessed via } \\
\text { distracted and non- } \\
\text { distracted } 10 \text {-meter } \\
\text { walk. [Time Frame: } \\
2 \text { years] }\end{array}$ & \\
\hline
\end{tabular}




\section{TABLE 1 Summary of Phase 3 Preclinical Alzheimer Disease Clinical Trials (continued)}

\begin{tabular}{|c|c|c|c|c|c|c|}
\hline $\begin{array}{l}\text { Clinical Trial } \\
\text { (estimated } \\
\text { enrollment) } \\
\text { [Sponsor] }\end{array}$ & $\begin{array}{c}\text { Drugs } \\
\text { [Drug Class/Type } \\
\text { (Mechanism of } \\
\text { Action)] }\end{array}$ & Inclusion & Exclusion & $\begin{array}{c}\text { Primary } \\
\text { Endpoint } \\
\text { [Measurement } \\
\text { Time Frame] }\end{array}$ & $\begin{array}{c}\text { Secondary } \\
\text { Endpoints } \\
\text { [Measurement } \\
\text { Time Frame] }\end{array}$ & $\begin{array}{c}\text { Start and } \\
\text { (estimated) } \\
\text { End Date }\end{array}$ \\
\hline & & & $\begin{array}{l}\text { 10. Uncontrolled diabetes mel- } \\
\text { litus, defined as hemoglobin } \\
\text { Alc }>7.5 \% \text {, or requiring insu- } \\
\text { lin treatment. } \\
\text { 11. Regularly smoking ciga- } \\
\text { rette within the past year. } \\
\text { 12. Women with a potential } \\
\text { for pregnancy, lactation/child } \\
\text { bearing (2-year post-meno- } \\
\text { pausal or surgically sterile to } \\
\text { be considered not child bear- } \\
\text { ing potential). } \\
\text { 13. Participant enrolled in } \\
\text { another investigational drug or } \\
\text { device study, either currently } \\
\text { or within the past } 2 \text { months. } \\
\text { 14. Severe obesity with } \\
\text { BMI } 455 \text {; clinical judgment } \\
\text { should be applied in all cases } \\
\text { to assess patient safety and } \\
\text { anticipated compliance. } \\
\text { 15. Allergy to angiotensin } \\
\text { receptor blockers (ARBs), i.e., } \\
\text { drugs that have a suffix } \\
\text { "-sartan." } \\
\text { 16. Allergy to other study } \\
\text { drugs or their ingredients; for } \\
\text { example, clinical history or } \\
\text { self-reported allergy or intoler- } \\
\text { ance to atorvastatin. } \\
\text { 17. Abnormal screening labo- } \\
\text { ratory tests (e.g. liver ALT and } \\
\text { AST } 3 \times \text { ULN, CK }>3 \times \text { ULN, } \\
\text { GFR } 30 \text { or Hct }<28 \% \text { ); may } \\
\text { be rescreened after } 2 \text { weeks or } \\
\text { longer. } \\
18 \text {. A medical condition likely } \\
\text { to limit survival to less than } \\
3 \text { years. } \\
\text { 19. Participant has any con- } \\
\text { ditions judged by the study } \\
\text { investigator to be medically } \\
\text { inappropriate, risky or likely } \\
\text { to cause poor study compli- } \\
\text { ance. For example: } \\
\text { a. Plans to move outside the } \\
\text { clinic catchment area in the } \\
\text { next } 2 \text { years. } \\
\text { b. Significant concerns about } \\
\text { participation in the study from } \\
\text { spouse, significant other, or } \\
\text { family members. } \\
\text { c. Lack of support from pri- } \\
\text { mary health care provider. } \\
\text { d. Residence too far from the } \\
\text { study clinic site such that trans- } \\
\text { portation is a barrier including } \\
\text { persons who require transporta- } \\
\text { tion assistance provided by the } \\
\text { study clinic funds for screening } \\
\text { or randomization visits. } \\
\text { e. Residence in a nursing } \\
\text { home; persons residing in an } \\
\text { assisted living or retirement } \\
\text { community are eligible if they } \\
\text { meet the other criteria. }\end{array}$ & & & \\
\hline
\end{tabular}




\section{TABLE 1 Summary of Phase 3 Preclinical Alzheimer Disease Clinical Trials (continued)}

\begin{tabular}{|c|c|c|c|c|c|c|}
\hline $\begin{array}{l}\text { Clinical Trial } \\
\text { (estimated } \\
\text { enrollment) } \\
\text { [Sponsor] }\end{array}$ & $\begin{array}{c}\text { Drugs } \\
\text { [Drug Class/Type } \\
\text { (Mechanism of } \\
\text { Action)] }\end{array}$ & Inclusion & Exclusion & $\begin{array}{c}\text { Primary } \\
\text { Endpoint } \\
\text { [Measurement } \\
\text { Time Frame] }\end{array}$ & $\begin{array}{l}\text { Secondary } \\
\text { Endpoints } \\
\text { [Measurement } \\
\text { Time Frame] }\end{array}$ & $\begin{array}{c}\text { Start and } \\
\text { (estimated) } \\
\text { End Date }\end{array}$ \\
\hline & & & $\begin{array}{l}\text { f. Other medical, psychiatric, } \\
\text { or behavioral factors that, in } \\
\text { the judgment of the site PI } \\
\text { or clinician, may interfere } \\
\text { with study participation or } \\
\text { the ability to follow the study } \\
\text { protocol. } \\
\text { g. Couples or significant part- } \\
\text { ners who live together cannot } \\
\text { be enrolled or participate } \\
\text { simultaneously in the study. } \\
\text { 20. Lack of approval from par- } \\
\text { ticipant's regular health care } \\
\text { providers, i.e., a signed letter } \\
\text { of agreement for the partici- } \\
\text { pants to be enrolled in rrAD. }\end{array}$ & & & \\
\hline \multicolumn{7}{|c|}{ Targeting Preclinical, Prodromal, or Mild Stages of AD } \\
\hline $\begin{array}{l}\text { NCT01760005a } \\
\text { ( } \mathrm{n}=490 \text { ) } \\
\text { [Washington } \\
\text { University School } \\
\text { of Medicine] }]^{22}\end{array}$ & $\begin{array}{l}\text { Gantenerumab, } \\
\text { solanezumab } \\
\text { [Monoclonal } \\
\text { antibody (anti- } \\
\text { amyloid)] }\end{array}$ & $\begin{array}{l}\text { Between } 18-80 \text { years of age } \\
\text { Individuals who know } \\
\text { they have an AD-causing } \\
\text { mutation or are unaware } \\
\text { of their genetic status and } \\
\text { have an autosomal domi- } \\
\text { nant Alzheimer disease } \\
\text { mutation in their family. } \\
\text { Are within -15 to + } 10 \text { years } \\
\text { of the predicted or actual } \\
\text { age of cognitive symptom } \\
\text { onset for treatment arms. } \\
\text { For Cognitive Run-In: } \\
\text { includes participants who } \\
\text { are younger than } 15 \text { years } \\
\text { prior to the expected age of } \\
\text { cognitive symptom onset, } \\
\text { in addition to those } 15 \\
\text { years younger and no more } \\
\text { than } 10 \text { years older than } \\
\text { expected or actual age of } \\
\text { cognitive symptom onset. } \\
\text { Cognitively normal or with } \\
\text { mild cognitive impairment } \\
\text { or mild dementia, Clinical } \\
\text { Dementia Rating (CDR) of } \\
\text { 0-1 (inclusive). } \\
\text { Fluency in DIAN-TU trial } \\
\text { approved language and evi- } \\
\text { dence of adequate premor- } \\
\text { bid intellectual functioning. } \\
\text { Able to undergo MRI, } \\
\text { Lumbar Puncture (LP), } \\
\text { PET, and complete all study } \\
\text { related testing and } \\
\text { evaluations. } \\
\text { For women of childbear- } \\
\text { ing potential, if partner } \\
\text { is not sterilized, subject } \\
\text { must agree to use effective } \\
\text { contraceptive measures } \\
\text { (hormonal contraception, } \\
\text { intra-uterine device, sexual } \\
\text { abstinence, barrier method } \\
\text { with spermicide). }\end{array}$ & $\begin{array}{l}\text { History or presence of brain } \\
\text { MRI scans indicative of any } \\
\text { other significant abnormality. } \\
\text { Alcohol or drug dependence } \\
\text { currently or within the past } \\
1 \text { year. } \\
\text { Presence of pacemakers, } \\
\text { aneurysm clips, artificial heart } \\
\text { valves, ear implants, or foreign } \\
\text { metal objects in the eyes, skin } \\
\text { or body which would preclude } \\
\text { MRI scan. } \\
\text { History or presence of clini- } \\
\text { cally significant cardiovas- } \\
\text { cular disease, hepatic/renal } \\
\text { disorders, infectious disease } \\
\text { or immune disorder, or meta- } \\
\text { bolic/endocrine disorders } \\
\text { Anticoagulants except low } \\
\text { dose ( } 325 \text { mg) aspirin. } \\
\text { Have been exposed to a mono- } \\
\text { clonal antibody targeting beta } \\
\text { amyloid peptide within the } \\
\text { past } 6 \text { months. } \\
\text { History of cancer within the } \\
\text { last } 5 \text { years, except basal cell } \\
\text { carcinoma, non-squamous } \\
\text { skin carcinoma, prostate can- } \\
\text { cer or carcinoma in situ with } \\
\text { no significant progression over } \\
\text { the past } 2 \text { years. } \\
\text { Positive urine or serum preg- } \\
\text { nancy test or plans or desires } \\
\text { to become pregnant during the } \\
\text { course of the trial. } \\
\text { Subjects unable to complete all } \\
\text { study related testing, including } \\
\text { implanted metal that cannot } \\
\text { be removed for MRI scanning, } \\
\text { required anticoagulation and } \\
\text { pregnancy. }\end{array}$ & $\begin{array}{l}\text { Assess cogni- } \\
\text { tive efficacy in } \\
\text { individuals with } \\
\text { mutations caus- } \\
\text { ing dominantly } \\
\text { inherited AD } \\
\text { as measured by } \\
\text { change in the } \\
\text { DIAN-TU cogni- } \\
\text { tive composite } \\
\text { score. [Time } \\
\text { Frame: Baseline } \\
\text { and Weeks 52, } \\
\text { 104, 156, and } \\
\text { 208] }\end{array}$ & $\begin{array}{l}\text { 1. Gantenerumab: } \\
\text { Cerebral amyloid } \\
\text { imaging using [11C] } \\
\text { PiB-PET. [Time } \\
\text { Frame: Baseline and } \\
\text { Weeks 52, 104, and } \\
\text { 208] } \\
\text { 2. Solanezumab: } \\
\text { Total Abeta 1-42 } \\
\text { (A } 342 \text { ) in CSF. [Time } \\
\text { Frame: Baseline, } \\
\text { Week 104 and Week } \\
\text { 208] } \\
\text { 3. Change from } \\
\text { Baseline in Clinical } \\
\text { Measures [Time } \\
\text { Frame: Baseline, } \\
\text { week 208]: } \\
\text { Clinical Dementia } \\
\text { Rating (CDR), } \\
\text { including CDR sum } \\
\text { of boxes (CDR-SB) } \\
\text { and clinician's diag- } \\
\text { nostic assessment } \\
\text { Geriatric Depression } \\
\text { Scale (GDS) } \\
\text { Neuropsychiatric } \\
\text { Inventory } \\
\text { Questionnaire } \\
\text { (NPI-Q) } \\
\text { Functional } \\
\text { Assessment Scale } \\
\text { (FAS) } \\
\text { Mini Mental Status } \\
\text { Exam (MMSE) } \\
\text { 4. Change from } \\
\text { Baseline in } \\
\text { Cognitive Measures } \\
\text { [Time Frame: } \\
\text { Baseline, week } \\
\text { 208]: International } \\
\text { Shopping List Test } \\
\text { (12-Item Word List } \\
\text { Learning): } 3 \text { learn- } \\
\text { ing trials, Immediate } \\
\text { Recall, 30-min } \\
\text { Delayed Recall } \\
\text { (CogState) } \\
\end{array}$ & $\begin{array}{l}\text { December } \\
2012 \text { \& } \\
\text { March } 2021\end{array}$ \\
\hline
\end{tabular}




\section{TABLE 1 Summary of Phase 3 Preclinical Alzheimer Disease Clinical Trials (continued)}

\begin{tabular}{|c|c|c|c|c|c|c|}
\hline $\begin{array}{l}\text { Clinical Trial } \\
\text { (estimated } \\
\text { enrollment) } \\
\text { [Sponsor] }\end{array}$ & $\begin{array}{c}\text { Drugs } \\
\text { [Drug Class/Type } \\
\text { (Mechanism of } \\
\text { Action)] }\end{array}$ & Inclusion & Exclusion & $\begin{array}{c}\text { Primary } \\
\text { Endpoint } \\
\text { [Measurement } \\
\text { Time Frame] }\end{array}$ & $\begin{array}{l}\text { Secondary } \\
\text { Endpoints } \\
\text { [Measurement } \\
\text { Time Frame] }\end{array}$ & $\begin{array}{c}\text { Start and } \\
\text { (estimated) } \\
\text { End Date }\end{array}$ \\
\hline & & $\begin{array}{l}\text { Adequate visual and audi- } \\
\text { tory abilities to perform all } \\
\text { aspects of the cognitive and } \\
\text { functional assessments. } \\
\text { Has a Study Partner who in } \\
\text { the investigator's judgment } \\
\text { is able to provide accurate } \\
\text { information as to the sub- } \\
\text { ject's cognitive and func- } \\
\text { tional abilities, who agrees } \\
\text { to provide information } \\
\text { at the study visits which } \\
\text { require informant input for } \\
\text { scale completion. }\end{array}$ & & & $\begin{array}{l}\text { Groton Maze } \\
\text { Learning Test: Timed } \\
\text { Chase Task, } 5 \text { learn- } \\
\text { ing Trials, Immediate } \\
\text { Recall, 30-min } \\
\text { Delayed Recall } \\
\text { (CogState) } \\
\text { Cogstate Detection } \\
\text { Task } \\
\text { Cogstate } \\
\text { Identification Test } \\
\text { Cogstate One Card } \\
\text { Learning Test } \\
\text { Cogstate One-Back } \\
\text { (OBK) Task } \\
\text { Behavioral Pattern } \\
\text { Separation Object } \\
\text { Task } \\
\text { Memory Complaint } \\
\text { Questionnaire } \\
\text { (MAC-Q) Trails } \\
\text { A \& B } \\
\text { Wechsler Memory } \\
\text { Scale - Revised } \\
\text { (WMS-R) Digit Span } \\
\text { Wechsler Adult } \\
\text { Intelligence Scale - } \\
\text { Revised (WAIS-R) } \\
\text { Digit-Symbol } \\
\text { Substitution Test } \\
\text { Raven's Progressive } \\
\text { Matrices (Set A) } \\
\text { Category Fluency } \\
\text { (Animals \& } \\
\text { Vegetables) } \\
\text { Wechsler Memory } \\
\text { Scale Logical } \\
\text { Memory I Paragraph } \\
\text { Memory (Immediate } \\
\text { \& Delayed Recall) }\end{array}$ & \\
\hline
\end{tabular}

a Phase 2/3.

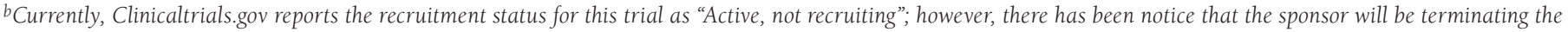
study (https://www.fiercebiotech.com/biotech/weak-data-spur-amgen-novartis-to-can-pivotal-alzheimer-s-test).

cPer study description.

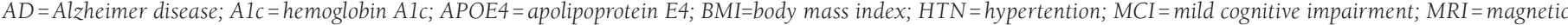
resonance imaging; PET = positron emission tomography; rrAD= risk reduction for Alzheimer disease; VA=Department of Veterans Affairs.

range from 2021 to 2025 (Table 1). ${ }^{16-22}$ Four of these 6 trials involve drugs that target amyloid (e.g., remove amyloid and/or prevent aggregation or production). The other 2 drugs aim for neuroprotection either directly or through improving cardiovascular health. ${ }^{16}$ One of the clinical trials is enrolling patients not only in the preclinical stage, but also in the MCI and mild dementia stages of AD. ${ }^{21}$

\section{Clinical Trial Populations}

The 6 clinical trials are enrolling patients at high risk for AD based on family history, presence of apolipoprotein E4 (apoE4) gene, or elevated brain amyloid through positron emission tomography (PET) scan or cerebrospinal fluid (CSF; Table 1).
All 6 clinical trials require that enrolled patients are cognitively unimpaired, with normal scores on tests such as the MiniMental State Examination. The 1 exception is a trial targeting not only preclinical AD, but also MCI and mild dementia.

Enrollment in most of these trials is restricted to relatively healthy patients, such as those without serious or unstable illness. Patients also cannot have a recent history (within the last few years) of cancer, serious brain infection, or head trauma and must not already be on an AD treatment. Enrollment generally excludes individuals with a recent history of mental health issues (e.g., suicide, depression, and bipolar disorder) or substance abuse problems. Because of the nature of certain endpoints, patients must be able to undergo 
magnetic resonance imaging (MRI) and, therefore, not have pacemakers or artificial heart valves, as well as be willing to undergo lumbar punctures (for collection of CSF). Patients must also be fluent in English and have adequate visual and auditory function to allow neuropsychological testing.

Five of the 6 clinical trials include patients aged under 65 years (Table 1), while 1 trial expands beyond the preclinical stage of $\mathrm{AD}$ and includes patients aged as young as 18 years. The most common age ranges are 50 or 60 years to 75 or 85 years.

\section{Clinical Trial Endpoints}

All but 1 trial has a primary endpoint related to cognitive function (Table 1). However, across the trials, cognitive function is measured through different tests, such as the Preclinical Alzheimer Cognitive Composite (PACC), Alzheimer's Prevention Initiative Composite Cognitive (APCC) Test, Dominantly Inherited Alzheimer Network Trials Unit (DIAN-TU) Cognitive Composite, and NIH Toolbox (NIH-TB) Cognition Battery. The majority of these endpoints (4 of 6 trials) were measured at baseline and again 4 or more years after start of treatment. However, some trials involved repeated measurements throughout the study period, and other primary endpoints were measured as time-to-event outcomes (Table 1). Additional primary endpoints include time to diagnosis of MCI or dementia because of AD. The 1 trial with a primary endpoint related to cerebral blood flow has a secondary endpoint related to cognitive function.

Common secondary endpoints include additional measures of cognitive function as reported in Table 1; biomarkers such as amyloid and tau levels and deposition, as measured through PET scans and/or CSF; other pathophysiologic changes associated with $\mathrm{AD}$, such as brain white matter and perfusion abnormalities as measured through MRIs; and functional measures such as from the Alzheimer's Disease Cooperative StudyActivities Daily Living-Prevention Questionnaire (Table 1).

The combination of cognition, function, and biomarker endpoints in the trials is aligned with the FDA's perspective on developing drugs to be used in the earlier stages of AD. According to draft guidance, the FDA would ideally like to see that a drug used in the earlier stages of AD has an effect on functional and cognitive deficits and suggests trials with sufficient duration to achieve this. ${ }^{23}$ Within the context of preclinical AD, the FDA draft guidance mentions that effects on characteristic pathophysiologic changes of $\mathrm{AD}$, or biomarkers, could serve as the basis for accelerated approval. ${ }^{23}$ However, at this time, there is insufficient evidence that an effect on biomarkers in $\mathrm{AD}$ will be reasonably likely to predict clinical benefit, which is the standard for accelerated approval. ${ }^{23}$

\section{Looking Ahead: Potential Coverage Considerations}

$\overline{\text { Although no drugs have been approved for the preclinical stage }}$ of $\mathrm{AD}$, review of the 6 ongoing phase 3 clinical trials raises potential issues of which payers should be aware. First, if a drug for the preclinical stage of AD is approved in high-risk patients, identifying such patients for treatment may require substantial cost and effort. ${ }^{24-26}$ Currently, preclinical AD trials are identifying patients at increased risk through family history and then genetic testing or PET imaging. Genetic testing and PET imaging can pose barriers to patient identification. Although some genetic testing prices are declining, the large number of patients who may be eligible to receive genetic testing could lead to a high budgetary impact for the payer. Additionally, genetic testing may require the involvement of genetic counselors, which would lead to additional costs and perhaps further barriers because genetic counselors are also limited in supply. Yet another barrier is patient rejection of genetic testing because of the fear of such possible negative consequences as higher premiums when purchasing long-term insurance if discovered to be at high risk of certain conditions.

Similarly, PET imaging can be expensive, and patients may not want to undergo such testing. As a result, identifying appropriate patients for treatment may require new initiatives around education, screening, and follow-up. Such initiatives may involve multiple stakeholders and be costly. For example, electronic medical record vendors could play a role in developing screening algorithms. Providers could then play a role in implementing the education, screening, and follow-up; however, they would need financial incentives to perform these services.

Other technologies on the horizon could also help with screening, such as blood tests and retina scans. However, these technologies are still being tested, and it remains unknown whether they will replace the use of PET imaging. ${ }^{27,28}$ If successful, a combination of these technologies alongside assessing family history, genetic testing, and PET testing could be used to develop an approach to screen patients at high risk for preclinical AD.

Second, the majority of clinical trials involve patients aged under 65 years and measure cognitive function 4 or more years later. If treatment is approved in a pre-Medicare population, but effects on cognition and function do not occur until many years later when patients have transitioned to another payer such as Medicare or Medicaid (for long-term care costs), some payers may be less willing to cover the medications. Therefore, there may be a need for alternative payment approaches or policy solutions to provide incentives for an earlier payer to cover medicines that may not increase health or delay costs until after the beneficiary has left the health plan. A similar problem ocurred with hepatitis $C$ virus treatments, and several payers reacted by implementing novel payment approaches. ${ }^{29,30}$

Third, past literature has suggested heterogeneity in AD progression and etiology. ${ }^{31,32}$ Several of the preclinical AD trials discussed in this article focus on populations with specific genetic variants or mutations (e.g., homozygous apoE4 
genotype, at least 1 apoE4 gene if heterozygote, and autosomal dominant AD mutation). This may result in drug treatments that are approved with narrow and differing indications. If there are major differences in the treatment effect for one indication versus another, payers may consider indication-based pricing contracts that vary payment based on the value associated with each indication, ${ }^{33,34}$ or other alternatives to volumebased contracts, such as a per-member per-month payment in a covered population. ${ }^{29,35}$

Fourth, the clinical trials are excluding sicker populations with serious or unstable illness, recent history of mental health or substance abuse conditions, and inability or unwillingness to undergo CSF draws, MRI scanning, and/or genetic testing. Thus, the clinical trials do not reflect the mix of high-risk patients seen in the real-world setting, and the treatment effects seen in the clinical trial populations may not be the same as in sicker populations. Since the first of these medications would likely be a novel drug class that is administered long term, the budgetary impact could be very high. Payers would have added pressure to ensure that this medication was cost-effective. Therefore, payers and manufacturers may want to consider an outcomes-based payment arrangement that includes monitoring of cognition and function in patients receiving and adhering to the treatment to ensure that the drug brings value. They may also consider coverage accompanied by the conduct of additional "real world" clinical studies that develop better evidence on benefits in these populations, as well as additional evidence (e.g., on longer-term outcomes and functional outcomes) relevant to the value of these treatments.

In addition, depending on the efficacy and safety evidence of the treatments, individuals at elevated risk who do not meet the specific risk measures included in the trials, as well as individuals with lower risk, may want "off-label" coverage because they too expect some benefits from treatment. These benefits may not be as large as for the specific highrisk patients targeted in the trials, requiring larger and potentially more costly long-term studies to determine safety and effectiveness. Mechanisms for developing such evidence, and for coverage and payment policy while such evidence is being developed, may also require innovative approaches by payers and manufacturers, such as coverage with evidence development or "adaptive" payment approaches that adjust payments as evidence accumulates. ${ }^{36}$

\section{Conclusions}

Disease-modifying drugs are being developed for use in the earlier stages of AD. The majority of ongoing, phase 3, preclinical AD trials include patients aged over a range of at least 60 to 75 years, with no cognitive impairment but at high risk of AD because of family history and specific genetic variations of AD or evidence of brain pathology through PET imaging. Most primary endpoints are related to cognition and are measured through a variety of tests at 4 or more years. Secondary endpoints include biomarkers, other measures of cognition, and measures of function, which is aligned with FDA draft guidance recommendations.

Review of these trials brings to light a few potential considerations when covering new medications in the future: (a) new and potentially costly approaches involving blood-based biomarker testing, genetic testing and/or PET imaging may be needed to identify appropriate patients and should be developed efficiently; (b) there may be a need for alternative payment approaches that encourage payers to incur costs that may have no short-term financial benefits but may result in longterm benefit for patients; (c) the value of AD treatments may differ across populations, creating a potential role for population-based contracting; and (d) payers and manufacturers may want to consider outcomes-based payment approaches and coverage with evidence development to mitigate uncertainty about the value of the treatment demonstrated in well-defined populations in clinical trials versus more heterogeneous realworld settings.

Given the magnitude of the potential health and cost consequences associated with these new early interventions to address $\mathrm{AD}$, further development of these approaches before the treatments come to market will enable payers, manufacturers, and policymakers to manage access and cost issues more effectively.

\section{Authors}

ANNA HUNG, PharmD, PhD, MS, Duke Clinical Research Institute, Durham, North Carolina. MONIKA SCHNEIDER, PhD; MARIANNE HAMILTON LOPEZ, PhD, MPA; and MARK MCCLELLAN, MD, PhD, Duke-Margolis Center for Health Policy, Washington, DC.

AUTHOR CORRESPONDENCE: Anna Hung, PharmD, PhD, MS, Postdoctoral Associate, Duke Clinical Research Institute, 200 Morris St., 7th Floor, Durham, NC 27701.

Tel.: 919.668.1493; E-mail: anna.hung@duke.edu.

\section{DISCLOSURES}

This work was funded through a generous gift from the Global CEO Initiative on Alzheimer Disease. Hung reports grants from Agency for Healthcare Research and Quality and Pharmaceutical Research and Manufacturers of America outside the submitted work and past employment at CVS Health and BlueCross BlueShield Association. McClellan is an independent board member on the boards of Johnson \& Johnson, Cigna, Alignment Healthcare, and Seer; co-chairs the Accountable Care Learning Collaborative and the Guiding Committee for the Health Care Payment Learning and Action Network; and receives fees for serving as an advisor for Cota and MITRE. Hamilton Lopez and Schneider have nothing to disclose.

Part of this work was presented at the 2019 AMCP Nexus Meeting, October 29-November 1, 2019, in National Harbor, MD. 


\section{ACKNOWLEDGMENT}

The authors thank the Advisory Group members involved with this work for their contributions to discussions on this topic and for the feedback provided.

\section{REFERENCES}

1. Masters CL, Bateman R, Blennow K, Rowe CC, Sperling RA, Cummings JL. Alzheimer's disease. Nat Rev Dis Primers. 2015;1:15056.

2. Hurd MD, Martorell P, Delavande A, Mullen KJ, Langa KM. Monetary costs of dementia in the United States. N Engl J Med. 2013;368(14):1326-34.

3. Aricept (donepezil hydrochloride) tablets. Eisai Inc. December 2018. Available at: https://www.accessdata.fda.gov/drugsatfda_docs/label/2018/02 0690s042,021720s014,022568s011lbl.pdf. Accessed May 18, 2020.

4. Exelon (rivastigmine tartrate) capsules. Novartis. December 2018. Available at: https://www.novartis.us/sites/www.novartis.us/files/exelon.pdf. Accessed May 18, 2020.

5. Razadyne (galantamine hydrobromide) tablets, for oral use. Janssen. February 2015. Available at: https://www.accessdata.fda.gov/drugsatfda_ docs/label/2015/021615s021lbl.pdf. Accessed May 18, 2020.

6. Namenda (memantine hydrochloride) tablets, for oral use. Allergan November 2018. Available at: https://media.allergan.com/actavis/actavis/ media/allergan-pdf-documents/product-prescribing/Final-Clean-NamendaUSPI.pdf. Accessed May 18, 2020

7. Alzheimer's Association. 2018 Alzheimer's disease facts and figures. Alzheimers Dement. 2018;14(3):367-429. Available at: https://doi. org/10.1016/j.jalz.2018.02.001. Accessed May 18, 2020.

8. Hansen RA, Gartlehner G, Webb AP, Morgan LC, Moore CG, Jonas DE. Efficacy and safety of donepezil, galantamine, and rivastigmine for the treatment of Alzheimer's disease: a systematic review and meta-analysis. Clin Interv Aging. 2008;3(2):211-25.

9. Casey DA, Antimisiaris D, O'Brien J. Drugs for Alzheimer's disease: are they effective? P T. 2010;35(4):208-11.

10. Alzheimer's Association. Research funding. Updated 2019. Available at: https://www.alz.org/get-involved-now/advocate/research-funding. Accessed May 18, 2020.

11. Sperling RA, Jack CR Jr, Aisen PS. Testing the right target and right drug at the right stage. Sci Transl Med. 2011;3(111):111 cm33. Available at: http://stm sciencemag.org/content/3/111/11lcm33.abstract. Accessed May 18, 2020.

12. Sperling RA, Aisen PS, Beckett LA, et al. Toward defining the preclinical stages of Alzheimer's disease: recommendations from the National Institute on Aging-Alzheimer's Association Workgroups on Diagnostic Guidelines for Alzheimer's Disease. Alzheimers Dement. 2011;7(3):280-92.

13. Albert MS, DeKosky ST, Dickson D, et al. The diagnosis of mild cognitive impairment due to Alzheimer's disease: recommendations from the National Institute on Aging-Alzheimer's Association Workgroups on Diagnostic Guidelines for Alzheimer's Disease. Alzheimers Dement $2011 \cdot 7(3): 270-79$

14. Jack CR Jr, Bennett DA, Blennow K, et al. NIA-AA research framework: toward a biological definition of Alzheimer's disease. Alzheimers Dement. 2018;14(4):535-62.

15. Langa KM, Levine DA. The diagnosis and management of mild cognitive impairment: a clinical review. JAMA. 2014;312(23):2551-61.

16. Cummings J, Lee G, Ritter A, Sabbagh M, Zhong K. Alzheimer's disease drug development pipeline: 2019. Alzheimers Dement (N Y). 2019;5:272-93.

17. Eli Lilly and Company. Clinical trial of solanezumab for older individuals who may be at risk for memory loss (A4). ClinicalTrials.gov Identifier: NCT02008357. Updated 2018. Retrieved November 17, 2019. Available at: https://clinicaltrials.gov/ct2/show/NCT02008357. Accessed May 18, 2020.
18. Novartis Pharmaceuticals. A study of CAD106 and CNP520 versus placebo in participants at risk for the onset of clinical symptoms of Alzheimer's disease (generation S1). ClinicalTrials.gov Identifier: NCT02565511. Updated 2019. Retrieved November 17, 2019. Available at: https://clinicaltrials.gov/ct2/show/NCT02565511. Accessed May 18, 2020.

19. Novartis Pharmaceuticals. A study of CNP520 versus placebo in participants at risk for the onset of clinical symptoms of Alzheimer's disease (generation S2). ClinicalTrials.gov Identifier: NCT03131453. Updated 2019. Retrieved November 17, 2019. Available at: https://clinicaltrials.gov/ct2/ show/NCT03131453. Accessed May 18, 2020.

20. VA Office of Research and Development. Brain amyloid and vascular effects of eicosapentaenoic acid (BRAVE-EPA). ClinicalTrials.gov Identifier: NCT02719327. Updated 2019. Retrieved November 17, 2019. Available at: https://clinicaltrials.gov/ct2/show/NCT02719327. Accessed May 18, 2020.

21. Washington University School of Medicine. Dominantly inherited alzheimer network trial: an opportunity to prevent dementia. A study of potential disease modifying treatments in individuals at risk for or with a type of early onset Alzheimer's disease caused by a genetic mutation (DIANTU). ClinicalTrials.gov Identifier: NCT01760005. Updated 2019. Retrieved November 17, 2019. Available at: https://clinicaltrials.gov/ct2/show/ NCT01760005. Accessed May 18, 2020.

22. University of Texas Southwestern Medical Center. Risk reduction for Alzheimer's disease (rrAD). ClinicalTrials.gov Identifier: NCT02913664. Updated 2019. Retrieved November 17, 2019. Available at: https:// clinicaltrials.gov/ct2/show/NCT02913664. Accessed May 18, 2020.

23. U.S. Food and Drug Administration. Alzheimer's disease: developing drugs for treatment. Guidance document. February 2018. Available at: https://www.fda.gov/regulatory-information/search-fda-guidance-documents/alzheimers-disease-developing-drugs-treatment-guidance-industy. Accessed May 18, 2020

24. Mitka M. PET imaging for Alzheimer disease: are its benefits worth the cost? JAMA. 2013;309(11):1099-100

25. Hornberger J, Bae J, Watson I, Johnston J, Happich M. Clinical and cost implications of amyloid beta detection with amyloid beta positron emission tomography imaging in early Alzheimer's disease - the case of florbetapir. Curr Med Res Opin. 2017;33(4):675-85.

26. Wittenberg R, Knapp M, Karagiannidou M, Dickson J, Schott J. Economic impacts of introducing diagnostics for mild cognitive impairment Alzheimer's disease patients. Alzheimers Dement (NY). 2019;5:382-87. Available at: https://www.ncbi.nlm.nih.gov/pmc/articles/PMC6709060/. Accessed May 18, 2020

27. Schindler SE, Bollinger JG, Ovod V, et al. High-precision plasma betaamyloid $42 / 40$ predicts current and future brain amyloidosis. Neurology. 2019;93(17):el647-e1659.

28. University of Rhode Island. Atlas of retinal imaging in Alzheimer's study (ARIAS). ClinicalTrials.gov Identifier: NCT03862222. Updated October 1, 2019. Available at: https://Clinicaltrials.gov/ct2/show/NCT03862222. Accessed May 18, 2020.

29. Trusheim MR, Cassidy WM, Bach PB. Alternative state-level financing for hepatitis C treatment—the "Netflix model". JAMA. 2018;320(19):1977-78.

30. Dan C. States innovate with payment models to expand access to hepatitis C treatment. U.S. Department of Health \& Human Services. October 29, 2019. Available at: https://www.hhs.gov/hepatitis/blog/2019/10/29/statesinnovate-with-payment-models-to-expand-access-to-hepatitis-c-treatment. html. Accessed May 18, 2020.

31. Au R, Piers RJ, Lancashire L. Back to the future: Alzheimer's disease heterogeneity revisited. Alzheimers Dement (Amst). 2015;1(3):368-70.

32. Komarova NL, Thalhauser CJ. High degree of heterogeneity in Alzheimer's disease progression patterns. PLoS Comput Biol. 2011;7(11):e1002251.

33. Bach PB. Indication-specific pricing for cancer drugs. JAMA. 2014;312 (16):1629-30. 
34. Express Scripts. Express Scripts data shows value-based programs deliver better health, affordability for costly conditions. March 27, 2019. Available at: https://www.express-scripts.com/corporate/articles/express-scriptsdata-shows-value-based-programs-deliver-better-health-affordability-costly. Accessed May 18, 2020.

35. Fiore N, Alexander M, Pothen M, et al. Novel state payment models for prescription drugs: early implementation successes and challenges. Duke Margolis Center for Health Policy and the Robert Wood Johnson Foundation. September 2019. Available at: https://Healthpolicy.duke.edu/sites/default/files/ u31/novel_state_payment_models_final_revised_091219.pdf. Accessed May 18, 2020
36. Daniel GW, Rubens EK, McClellan M. Coverage with evidence development for Medicare beneficiaries: challenges and next steps. JAMA Intern Med. 2013;173(14):1281-82. 